\title{
PERSPECTIVES
}

TIMELINE

\section{Guillain-Barré syndrome: a century of progress}

John A. Goodfellow and Hugh J. Willison

Abstract | In 1916, Guillain, Barré and Strohl reported on two cases of acute flaccid paralysis with high cerebrospinal fluid protein levels and normal cell counts novel findings that identified the disease we now know as Guillain-Barré syndrome (GBS). 100 years on, we have made great progress with the clinical and pathological characterization of GBS. Early clinicopathological and animal studies indicated that GBS was an immune-mediated demyelinating disorder, and that severe GBS could result in secondary axonal injury; the current treatments of plasma exchange and intravenous immunoglobulin, which were developed in the 1980s, are based on this premise. Subsequent work has, however, shown that primary axonal injury can be the underlying disease. The association of Campylobacter jejuni strains has led to confirmation that anti-ganglioside antibodies are pathogenic and that axonal GBS involves an antibody and complement-mediated disruption of nodes of Ranvier, neuromuscular junctions and other neuronal and glial membranes. Now, ongoing clinical trials of the complement inhibitor eculizumab are the first targeted immunotherapy in GBS.

Guillain-Barré syndrome (GBS) is the most common cause of acute flaccid paralysis, and manifests as rapidly evolving weakness and sensory disturbance in the arms, legs and, in some patients, facial, bulbar and respiratory muscles. Many patients make a good recovery over the months following symptom onset, but in severe cases patients can require months of intensive care and be left with permanent severe weakness, sensory disturbance and pain. Furthermore, $5 \%$ of patients die from complications, including respiratory failure, pneumonia and arrhythmias, making GBS a medical emergency with a high morbidity and significant mortality.

Descriptions of clinical cases that closely resemble the condition we now know as GBS were made at least as early as 1859 , when Jean Baptiste Octave Landry reported on "acute ascending paralysis". His report led to use of the term "Landry's ascending paralysis" to describe subacute ascending peripheral sensory and motor dysfunction. He observed the following:
The sensory and motor systems may be equally affected. However the main problem is usually a motor disorder characterised by a gradual diminution of muscular strength with flaccid limbs without contractures... The paralysis moves rapidly from lower to upper areas. The progression can be more or less rapid. When the paralysis reaches its maximum intensity, the danger of asphyxia is always imminent. However in eight out of ten cases death was avoided. When there is a reversal of the paralysis, the recovery period involves phenomena opposite to those indicated in the development period. Patients then either recover very quickly, or the disease becomes chronic with slow improvement (REF. 1).

Thus, the core clinical features of the condition were described, but its aetiology and pathogenesis remained obscure to mid-nineteenth and early twentieth century neurologists. The focus was on delineating the clinical features of this disorder to enable its differentiation from similar conditions, such as polio, which was a highly prevalent differential diagnosis for acute flaccid weakness. It was not until 1916 that Guillain, Barré and Strohl published the paper that would define the disease and the next 100 years of research.

\section{6 - Guillain, Barré and Strohl}

In 1916, Europe was on the brink of destruction, the Battle of the Somme had killed or wounded over one million men, yet Guillain, Barré and Strohl — three army physicians at the neurological military centre of the French Sixth Army - were discussing the cerebrospinal fluid (CSF) constituents and tendon reflexes of two paralysed soldiers ${ }^{2}$. In 1891, Walter Essex Wynter had published the first use of lumbar CSF sampling with a cutdown technique, and in the same year, Quincke reported the first use of the lumbar puncture $^{3}$. In 1916, Guillain, Barré and Strohl used Quincke's method to determine the protein level and cell count in the CSF of their patients.

The three neurologists observed high CSF protein levels in the absence of any rise in levels of inflammatory cells - their so-called "dissociation albumino-cytologique". This finding was distinct from the high white cell counts seen in the CSF of patients with other prevalent causes of acute flaccid paralysis, such as syphilis or polio. At the time, the finding firmly established that the condition was a clinical and pathological entity distinct from other infective causes of flaccid paralysis - Guillain-Barré syndrome was born.

Our current understanding of GBS allows for the finding of normal CSF protein levels, especially early in the disease, because we know that the CSF protein level might not be elevated until the second week, and for CSF white cell counts up to 50 cells/ $\mu$ l. Moreover, we also now consider a wide clinical spectrum to result from the same underlying acute immune-mediated peripheral nerve and nerve root inflammation. Nevertheless, the original CSF findings remain the CSF hallmark and a diagnostically important and supportive test result ${ }^{4}$.

\section{Early controversy}

Initially, the awkward eponym LandryGuillain-Barré-Strohl syndrome was 
used to describe the condition. By 1927, the term had been simplified to GuillainBarré syndrome ${ }^{5}$, even though Strohl had been instrumental in the electrographical recordings and characterization of the loss of tendon reflexes. Guillain and Barré continued as highly regarded neurologists after the war. Guillain insisted that the condition he described was benign, in the sense that it was not fatal, and therefore resisted the notion that Landry had described the same syndrome. Furthermore, he and Barré insisted on the high CSF protein level and low white cell count being necessary to define their syndrome and differentiate it from other infectious diseases. The clinical features compatible with the diagnosis were the subject of much debate; for example, the original report described no cranial nerve or bladder involvement, but with time, Guillain and Barré accepted these as possible features.

\section{Early pathological and animal studies}

In 1949, while debate continued about the nosological limits and essential clinical features of GBS, Haymaker and Kernohan published a large case series of autopsy findings from 50 patients with $\mathrm{GBS}^{6}$. This work remains one of the largest and most comprehensive pathological studies of GBS, although up to a quarter of patients included would not satisfy current definitions of GBS (for example some had CSF cell counts $>100$ cells/ $\mu \mathrm{l})$. In addition, the use of autopsy samples inevitably leads to selection for the most severely affected patients. Nonetheless, this study was influential because it set forth a concept of the pathogenesis of GBS. The authors reported nerve oedema during the first few days after onset, with subsequent focal swelling of myelin sheaths and irregularity of axons. They described lymphocytic and macrophagic infiltration from around day nine, and Schwann cell proliferation a few days later. They proposed that the cellular response was not the primary effector of nerve destruction, but secondary to an initial injury, which might be humorally mediated. Their proposal was the conceptual ancestor of the modern hypothesis: initial antibody-mediated injury with subsequent cellular infiltration, further damage, then repair.

This proposal of the pathogenesis had barely been articulated when, in 1955, an animal model of immune-mediated peripheral nerve inflammation experimental allergic neuritis (EAN) — was published ${ }^{7}$. In this study, rabbits developed weakness and ataxia $\sim 2$ weeks after immunization with sciatic nerve homogenates in Freund adjuvant (emulsified in mineral oil). The animals exhibited high CSF protein levels, normal white cell counts and lesions in the nerve roots, spinal ganglia and peripheral nerves. These lesions were characterized by perivascular infiltration with mononuclear cells, segmental demyelination and some axonal degeneration. The disease could be transferred to naive animals by injection of lymphocytes from immunized animals ${ }^{8}$. This work represented a breakthrough in animal modelling of autoimmune neuropathy, and enabled systematic unravelling of cellular and molecular mechanisms behind T-cell-mediated peripheral nerve demyelination. Subsequent work identified the myelin protein P2 as the neuritogenic factor ${ }^{9,10}$, showed that $\mathrm{P} 2$-specific $\mathrm{CD} 4{ }^{+}$cells are the main effector cells ${ }^{10}$, and revealed the potential contribution of autoantibodies ${ }^{11}$. EAN has proven to be a fruitful experimental model, and remains widely used.

The pathological features in the original report of EAN were at odds with the model of pathogenesis described by Haymaker and Kernohan. This discrepancy was addressed by an autopsy study of 19 patients with GBS, conducted at the institution at which the EAN model had been developed ${ }^{12}$. This study revealed a temporal and pathological profile that closely resembled EAN. Even in the earliest stages, marked lymphocytic and polymorphonuclear infiltrates were seen in peripheral and cranial nerves, and even in some terminal motor nerve branches. Myelin breakdown was seen in motor and sensory nerves, and there was retraction of myelin at nodes of Ranvier, leading to focal demyelination. Nerve roots were sometimes involved, and nerve injury was so proximal in some cases that changes were seen in the anterior horn cells. Patients who had survived the longest exhibited muscle denervation. The authors concluded that GBS is a "cell-mediated immunologic disorder, in which peripheral myelin is attacked by specifically-sensitized lymphocytes". Subsequent pathological studies confirmed these findings ${ }^{13}$ and extended the observations to identify the role of macrophages as major effectors of myelin stripping ${ }^{14}$ and axonal injury ${ }^{15}$.

The discrepancies between the report from Haymaker and Kernohan, which indicated minimal inflammation in the early pathological phase, and studies that have implicated T cells and macrophages as the main effectors of pathology remain somewhat unexplained. A pathological spectrum or dichotomy undoubtedly exists, with demyelination at one end and axonal injury at the other; Haymaker and Kernohan might have included a large proportion of patients with axonal injury and minimal cellular infiltration. Despite the discrepancy, human pathological data combined with an accessible animal model resulted in wide acceptance that the dominant pathological process is nerve demyelination mediated by T cells and macrophages, a model that has shaped our understanding of the disease for over 40 years.

\section{Charles Miller Fisher}

In 1956, Charles Miller Fisher reported three patient case histories in the New England Journal of Medicine ${ }^{16}$; little could he have known of the long-lasting impact these reports would have on our understanding of GBS. These patients had a triad of areflexia, ophthalmoplegia and ataxia, and Fisher proposed that they had an unusual variant of "idiopathic polyneuritis". The subacute onset and resolution of symptoms, along with the finding of albuminocytological dissociation, might have been what led him to consider the condition to be a variant of GBS with "an unusual and unique disturbance of peripheral neurons”.

Fisher's report is a masterpiece of the classic clinicoanatomical method of 19th and 20th century descriptive neurology, all the more remarkable because he saw one of the patients much later than the illness and drew his conclusions from their account and the clinical record. He identified signs that the CNS could be involved, which ultimately led to the realization that Miller Fisher syndrome (MFS), Bickerstaff brainstem encephalitis and GBS represent different points on the same immunopathological spectrum ${ }^{17}$.

\section{Inadvertent insights Association with swine flu vaccination} The epidemiology of GBS has been studied extensively, largely as a consequence of its reported association with the swine flu vaccination in 1976. Military recruits at Fort Dix in New Jersey, USA, had contracted an influenza-like illness, and several isolates from these patients were identified as $\mathrm{H} 1 \mathrm{~N} 1$ viruses that were antigenically similar to those that caused the great swine flu pandemic of 1918. Concern over another deadly pandemic led to production of enough vaccine to immunize the entire US population. 45 million people received the vaccine in October 1976. By December, 
Table 1 | Brighton criteria for Guillain-Barré syndrome

\begin{tabular}{|c|c|c|c|c|}
\hline \multirow[b]{2}{*}{ Diagnostic criteria } & \multicolumn{4}{|c|}{ Level of diagnostic certainty } \\
\hline & 1 & 2 & 3 & 4 \\
\hline Bilateral and flaccid weakness of limbs & + & + & + & $+1-$ \\
\hline $\begin{array}{l}\text { Decreased or absent deep tendon reflexes in } \\
\text { weak limbs }\end{array}$ & + & + & + & $+1-$ \\
\hline $\begin{array}{l}\text { Monophasic course and time between } \\
\text { onset-nadir } 12 \mathrm{~h} \text { to } 28 \text { days }\end{array}$ & + & + & + & $+1-$ \\
\hline CSF cell count $<50 / \mu \mathrm{l}$ & + & $+^{\star}$ & - & $+1-$ \\
\hline CSF protein concentration $>$ normal value & + & $+/-^{*}$ & - & $+1-$ \\
\hline $\begin{array}{l}\text { NCS findings consistent with one of the } \\
\text { subtypes of GBS }\end{array}$ & + & $+/-$ & - & $+1-$ \\
\hline Absence of alternative diagnosis for weakness & + & + & + & + \\
\hline \multicolumn{5}{|c|}{$\begin{array}{l}\text { +, present; -, absent; +/-, present or absent; GBS, Guillain-Barré syndrome; NCS, nerve conduction studies. } \\
\text { *If CSF is not collected or results not available, nerve electrophysiology results must be consistent with the } \\
\text { diagnosis of Guillain-Barre syndrome. Level } 1 \text { is the highest level of diagnostic certainty, level } 4 \text { is the lowest } \\
\text { level of diagnostic certainty. Reproduced with permission from Oxford University Press } \odot \text { Fokke, C. et al. } \\
\text { Brain 137, 33-43 (2014). }\end{array}$} \\
\hline
\end{tabular}

planned surveillance had identified clusters of GBS cases, leading to the conclusion that the risk of GBS was increased in the first 6 weeks after immunization (RR 7.6, 95\% CI 6.7-8.6). The immunization program was suspended by mid-December, but continued surveillance led to the conclusion that many cases of GBS were directly related to the vaccine ${ }^{18}$.

This report has caused nearly 40 years of concern and debate about the relationship of influenza vaccination - and, by analogy, all vaccines - with GBS. The report was questioned on several methodological issues, and an independent review of the GBS cases that were included noted that many did not meet the accepted diagnostic criteria - the original study consequently received fierce criticism ${ }^{19}$. The US Justice Department eventually convened an independent assessment of the data by an expert panel, who confirmed that, despite methodological flaws of the study, the risk of GBS was elevated and peaked 2-3 weeks after vaccination, with an attributable risk of 0.49-0.59 extra cases per 100,000 adult vaccinees ${ }^{20}$. Several subsequent influenza immunization programmes have been monitored for an increased incidence of GBS; in 1998, a retrospective case series of patients with GBS confirmed an increased risk of GBS (RR 1.7, 95\% CI 1.0-2.8) in the 6 weeks after influenza vaccination ${ }^{21}$.

Following the controversy precipitated by the 1976 swine flu vaccination, the Centers for Disease Control and Protection urged the National Institute of Neurological and Communicative Disorders and Stroke to develop more-stringent diagnostic criteria for GBS to ensure more definitive case ascertainment during monitoring. Diagnostic criteria had previously been proposed in 1960 (REF. 22), but the vaccination controversy led to development of the Asbury criteria ${ }^{23}$, which were further clarified in 1981 (REF. 24) and 1990 (REF. 25), largely to incorporate advances in neurophysiological techniques. These criteria served as the diagnostic standard for many clinical trials and epidemiological and pathological studies undertaken since their development. In 2014, the Brighton Collaboration (an international collaboration that facilitates the development of internationally standardized case definitions for various illnesses) developed case definition criteria (TABLE 1); use of these criteria and their validation in GBS cohorts has begun ${ }^{4}$.

\section{Association with gangliosides}

In the 1970s and 1980s, purified gangliosides were administered to patients in many countries for various neurological disorders. Gangliosides are sialylated glycosphingolipids that are enriched in neuronal membranes and involved in neuronal processes including synaptogenesis, neuritogenesis, neuronal precursor migration, neuronal regeneration and myelination ${ }^{26}$. Clinical trials had suggested that exogenous gangliosides improved symptoms of diabetic neuropathy and offered some neuroprotection in ischaemic stroke. Promising results had also been seen in the prevention and treatment of neurodegenerative diseases ${ }^{27}$.

In the 1990s, the role of anti-ganglioside antibodies in mediating peripheral nerve injury in GBS was becoming clearer (see Anti-ganglioside antibodies, below), and case reports emerged of GBS in patients who had received ganglioside-based medications ${ }^{28}$, triggering fears that these drugs were causing GBS in some patients. Eventually, most countries withdrew gangliosides for most indications, although controversy over their association with GBS persists. Several epidemiological studies have indicated no elevated risk of GBS in patients treated with gangliosides ${ }^{29,30}$, and no cases of GBS have been reported in several trials of the ganglioside GM1 for spinal cord injury ${ }^{31}$.

\section{Electrophysiological developments}

Early electrophysiological studies established that conduction block in peripheral nerves is the neurophysiological hallmark of GBS, and that this feature is part of a widespread, multifocal demyelinating process in most patients $^{32}$. These clinical findings were confirmed by many animal studies in which serum from EAN mice or from humans with GBS induced acute lesions with conduction block in wild-type mice, and passive transfer of serum from animals immunized with galactocerebroside or anti-galactocerebroside antibodies caused focal paranodal disruption and subsequent demyelination ${ }^{33-35}$.

These studies helped to clarify the nature of the underlying pathological process, and others focused on the diagnostic utility of electrophysiology. This work established that reduced motor amplitudes are the earliest changes in GBS, and that serial testing detects evolving demyelinating features ${ }^{36,37}$. These findings remain the cornerstone of electrodiagnosis in GBS, although the subsequently identified pure axonal form of GBS (see Axonal GBS, below) has different characteristic electrophysiological features: reduced compound muscle action potential amplitude and reversible conduction failure, among others. In early GBS, electrophysiological findings are often normal or difficult to classify, and can lead to misclassification of axonal forms as acute inflammatory demyelinating polyneuropathy (AIDP). Increasing recognition of this difficulty has resulted in calls for redefining the electrophysiological diagnostic criteria for $\mathrm{GBS}^{38}$.

\section{Axonal GBS}

By the mid-1980s, GBS was considered to be a primary demyelinating T-cell-mediated autoimmune disorder of peripheral nerves and nerve roots. Secondary axonal degeneration and Wallerian-like degeneration after severe demyelination was felt to represent the severe end of the 

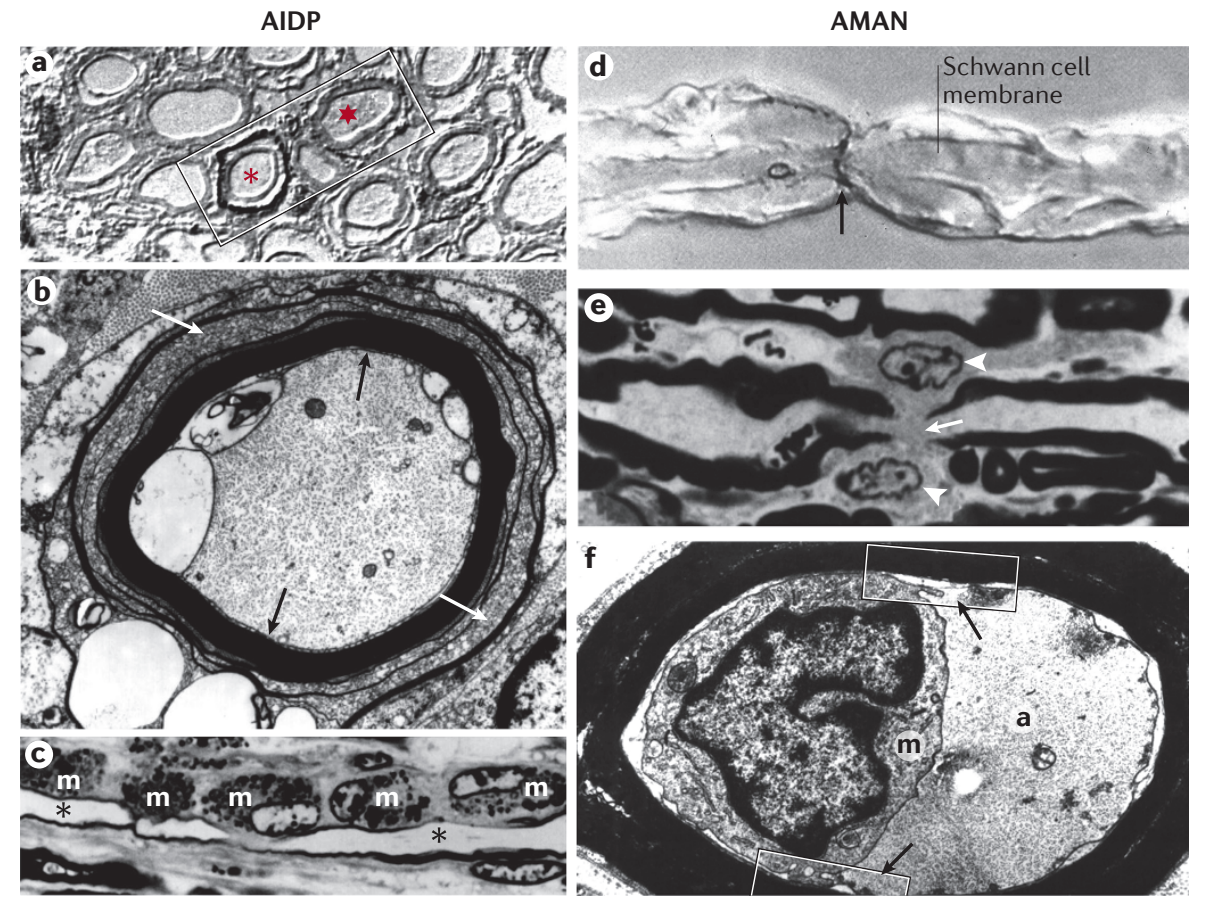

Figure 1 | Immune-mediated attack on Schwann cells and axons in Guillain-Barré syndrome. a | Deposition of the complement product C3d (black) on the outer surface of two fibres (asterisks) from a patient with autoimmune demyelinating acute inflammatory demyelinating polyneuropathy (AIDP). $\mathbf{b}$ | Extensive vesiculation of the myelin sheath in the same patient with AIDP (black arrows indicate normal compacted myelin; white arrows indicate abnormal vesiculation of myelin). c| Macrophages containing fatty myelin debris are associated with a demyelinated fibre from the same patient with AIDP, highlighting the central role inflammatory cells play in the pathogenesis of AIDP. (Asterisks label the axon; macrophages labelled with $\mathrm{m}$ ). $\mathbf{d} \mid \mathrm{C} 3 \mathrm{~d}$ deposition (arrow) at the node of Ranvier in the ventral root in a patient with acute motor axon neuropathy (AMAN). e | Node of Ranvier (arrow) on the ventral root, with visible nodal lengthening and two overlying macrophages (arrowheads) in a patient with AMAN. $\mathbf{f} \mid$ A macrophage $(\mathrm{m})$ in the internodal axon (a) beginning to extend processes towards the periaxonal space (black arrows). Parts $\mathbf{a}, \mathbf{b}$ and $\mathbf{c}$ modified with permission from John Wiley and Sons @ Hafer-Macko, C. E. et al. Ann. Neurol. 39, 625-635 (1996). Part d modified with permission from Annual Reviews (C) Ho, T. W. et al. Annu. Rev. Neurosci. 21, 187-226 (1998). Parts e and f modified with permission from Springer (c) Griffin, J. W. et al. J. Neurocytol. 25, 33-51 (1996).

demyelinating process. In 1985, however, Thomas Feasby and colleagues cared for a patient with severe post-diarrhoeal GBS who became tetraplegic and developed respiratory failure within $36 \mathrm{~h}$. Nerve conduction studies revealed inexcitable nerves - an unusual finding - and she died from a cardiac arrest on day 28 after onset of weakness. The autopsy revealed severe, widespread axonal degeneration in the nerve roots and periphery without lymphocytic inflammation, rather than the usual characteristic segmental demyelination. The team were convinced they had seen something new and published their findings from several similar cases in 1986 (REF. 39). Their proposal of a pure axonal form of GBS met with much scepticism, and Feasby had to fiercely defend his finding $\mathrm{s}^{40}$ until similar cases were reported ${ }^{41}$ and the concept gained some ground by the early 1990 s.
At a similar time, a collaborative group reported on a unique GBS-like syndrome - the Chinese paralytic syndrome (CPS) - that occurred in seasonal outbreaks among children in rural China ${ }^{42}$ and was characterized by severe flaccid tetraplegia that progressed rapidly and often necessitated ventilator support. Most patients exhibited high CSF protein levels and low or absent white cell counts, typical of GBS. Electrophysiological recordings of reduced motor amplitudes, preserved conduction velocities and preserved sensory nerve action potentials, however, pointed to a severe axonal neuropathy ${ }^{43}$. Autopsy tissue from 12 patients did not show demyelination typical of GBS, but extensive Wallerian-like degeneration of sensory and/or motor axons. The condition was classified as a type of GBS and subdivided according to the axons affected; the terms acute motor and sensory axonal neuropathy (AMSAN) and acute motor axonal neuropathy (AMAN) were coined. The pathology identified in this work - macrophage invasion of the periaxonal space at the paranodal and nodal regions, where there is immunoglobulin and complement deposition, and displacement of the axon ${ }^{44}-$ is in marked contrast to the macrophage-mediated myelin stripping and the antibody and complement deposition in Schwann cells in the demyelinating form of GBS ${ }^{45,46}$ (FIG. 1), which became known as acute inflammatory demyelinating polyneuropathy (AIDP) to reflect the pathological distinction from AMAN and AMSAN. Subsequent studies confirmed the existence of AMAN and AMSAN in populations globally, although the proportions of patients with GBS subtypes vary geographically; for example, AMAN and AMSAN are more common in China and Asia, whereas AIDP is more common in North America and Europe.

\section{Molecular mimicry in pathogenesis Campylobacter jejuni}

Long-standing recognition that a diarrhoeal illness can precede GBS has led many to search for an infectious trigger. For example, in 1958, Campbell reported antecedent respiratory tract infections in $60 \%$ of patients with GBS in his case series, and diarrhoea in $10-20 \%{ }^{47}$. He suggested that "the Landry-Guillain-Barré syndrome is a nonspecific reaction to several infective agents and is possibly due to an abnormal antigen-antibody response".

In 1982, Rhodes and Tattersfield reported on a patient with GBS after a diarrhoeal illness and whose stool was positive for Campylobacter jejuni $i^{48}$. This report was soon followed by a retrospective study of 56 patients with GBS, $38 \%$ of whom exhibited serological evidence of $C$. jejuni infection ${ }^{49}$. Numerous subsequent studies documented the prevalence of C. jejuni infection or seropositivity in patients with GBS, confirming the finding to be widespread and reproducible ${ }^{50}$.

In 1993, Yuki and colleagues demonstrated that the core lipo-oligosaccharides of the $C$. jejuni strains associated with GBS have structural similarity with various gangliosides present on peripheral nerve membranes $^{51}$, indicating that molecular mimicry of gangliosides could contribute to GBS. Furthermore, lipo-oligosaccharides from the $C$. jejuni strains associated with MFS were shown to have identical structures to that of ganglioside GQ1b ${ }^{52}$. In 2004, Yuki demonstrated that immunization of rabbits with the ganglioside-like 
lipo-oligosaccharides from GBS-associated C. jejuni strains resulted in a subacute flaccid tetraplegia and pathological changes similar to those seen in $\mathrm{AMAN}^{53}$.

\section{Anti-ganglioside antibodies}

In recent decades, the spotlight has fallen on the pathogenic mechanism that links C. jejuni and molecular mimicry of gangliosides with GBS. The presence of anti-GM1 ganglioside IgM antibodies in multifocal motor neuropathy has long been known, and other anti-ganglioside antibodies were reported in patients with GBS in 1988 (REF. 54). In 1990, Yuki postulated that anti-ganglioside antibodies might be present in a patient with AMAN following C. jejuni enteritis; he identified high titres of anti-GM1 IgG, which fell with resolution of the illness ${ }^{41}$. Numerous subsequent studies established that anti-GM1 IgG are present in a high proportion of patients with GBS, mostly those with AMAN or AMSAN ${ }^{55}$. Other anti-ganglioside antibodies were subsequently associated with specific clinical subtypes of GBS, including anti-GD1a antibodies with AMAN $^{56}$ and anti-GQ1b and anti-GT1a with acute oropharyngeal palsy ${ }^{57}$.

In the context of anti-ganglioside antibodies, the condition previously described by Miller Fisher took on greater significance. The association between specific anti-ganglioside antibodies and specific GBS subtypes became accepted, but the proportion of patients who were seropositive for these antibodies was generally relatively low and of limited diagnostic use. By contrast, in 1992 Chiba and colleagues detected anti-GQ1b IgG antibodies in six consecutive patients with MFS, and consequently proposed that it could be a useful diagnostic marker ${ }^{58}$. This report opened the door to a plethora of studies that confirmed that the presence of anti-GQ1b IgG antibodies in 80-95\% of patients with MFS and in many patients with MFS-GBS overlap conditions or Bickerstaff encephalitis ${ }^{17}$. Anti-GQ1b IgG antibodies have, therefore, served as a useful clinical diagnostic marker of MFS.

A remarkable twist in the anti-ganglioside antibody story has emerged with the discovery that some anti-ganglioside antibodies can only be detected in the blood of some patients with techniques that involve simultaneous presentation of multiple gangliosides or gangliosides with other lipids ${ }^{59,60}$. This observation has led to recognition of the fact that specific epitopes present in vivo might depend on interactions between multiple molecules on the cell membrane, and that this complex arrangement is not readily reproduced with traditional antibody testing methods $\mathrm{s}^{61,62}$. Novel diagnostic techniques have been developed to begin addressing this issue, and might reveal antibodies in patients who have hitherto tested seronegative ${ }^{63}$.

\section{Pathogenic mechanism}

The association of anti-ganglioside antibodies with specific GBS subtypes might, of course, be an epiphenomenon rather than an indication that the antibodies mediate pathogenesis. Numerous investigators over several decades and several continents have attempted to determine whether these antibodies are pathogenetic and how they mediate damage. In summary, many collaborative efforts have demonstrated that anti-GM1 and anti-GQ1b antibodies bind to peripheral nerve and neuromuscular junctions ${ }^{64,65}$, and anti-GD1a antibodies bind to the nodes of Ranvier, paranodal myelin and neuromuscular junction ${ }^{66-68}$. Upon binding, the antibodies activate the complement cascade, resulting in formation of the membrane attack complex, disruption of sodium channel clusters at the node of Ranvier with disruption of nodal architecture ${ }^{69}$, and calcium influx and calpain-dependent neuronal and glial injury at the neuromuscular junction ${ }^{70,71}$. This injury can be ameliorated with complement inhibitors ${ }^{72,73}$.

Taken together, these studies have revealed a likely pathogenic pathway for C. jejuni-associated GBS, at least in AMAN (FIG. 2). The relationship between anti-ganglioside antibodies and AIDP is not as clear because less evidence indicates associations of anti-ganglioside antibodies with this form of GBS. Notable exceptions, however, include evidence of molecular mimicry between Mycoplasma pneumoniae, production of anti-galactocerebroside antibodies and $\mathrm{AIDP}^{74}$.

\section{GBS and Zika virus}

Various viruses can trigger GBS, and in 2016, it became clear that those viral triggers include Zika virus. Since the 1950s, Zika virus has been reported as circulating sporadically in Africa and Southeast Asia, but in 2016, the Zika virus epidemic was declared a Public Health Emergency of International Concern by the WHO owing to rapid spread of the virus across Central and South America and elsewhere. Numerous neurological complications have been associated with Zika virus infection, and a case-control study from a localized outbreak in French Polynesia in 2013-2014 demonstrated an association and likely causative relationship between Zika virus infection and $\mathrm{GBS}^{75}$. The estimated incidence was 0.24 cases of GBS per 1,000 Zika virus infections; although a rare complication, in the context of an emerging epidemic or pandemic, this incidence of GBS could easily overwhelm the hospital and intensive care resources of even the best-equipped and well prepared of healthcare services. Additional case-control studies are emerging from other geographical areas, notably in Latin America, that are also reporting a very high incidence of Zika-associated GBS ${ }^{76-78}$. It therefore seems inevitable that this emergent form of GBS will continue to occur throughout Zika-affected regions in Asia and Africa.

\section{Therapy}

In parallel with all the aforementioned studies, a small number of trials have established the current standard treatments for GBS. In combination with meticulous medical and intensive care and long-term rehabilitation therapy, plasma exchange and intravenous immunoglobulin (IVIg) have been shown to improve outcomes for patients with GBS.

In 1984, two small clinical trials showed some positive effects of plasma exchange in $\mathrm{GBS}^{79,80}$, and in 1985, a larger study confirmed the effect and demonstrated that plasma exchange in patients who were unable to walk hastened recovery, especially when started within 2 weeks of GBS onset ${ }^{81}$. A subsequent study obtained similar results ${ }^{82}$. Plasma exchange consequently became the first proven therapy for GBS, but the treatment is not always straightforward and carries particular risks for patients with autonomic disturbance, which is common in GBS.

Largely owing to difficulties in performing plasma exchange in the late 1980s, a group in the Netherlands sought an alternative therapy and used fresh frozen plasma and then, in subsequent patients, IVIg for GBS or chronic inflammatory demyelinating polyneuropathy ${ }^{83-85}$. This work led to a clinical trial in the late 1980s in which plasma exchange and IVIg were compared $^{86}$. The results showed that IVIg was a practical and effective alternative to plasma exchange: the proportion of patients who showed improvement after 4 weeks was higher among patients who received IVIg than among those who received plasma 


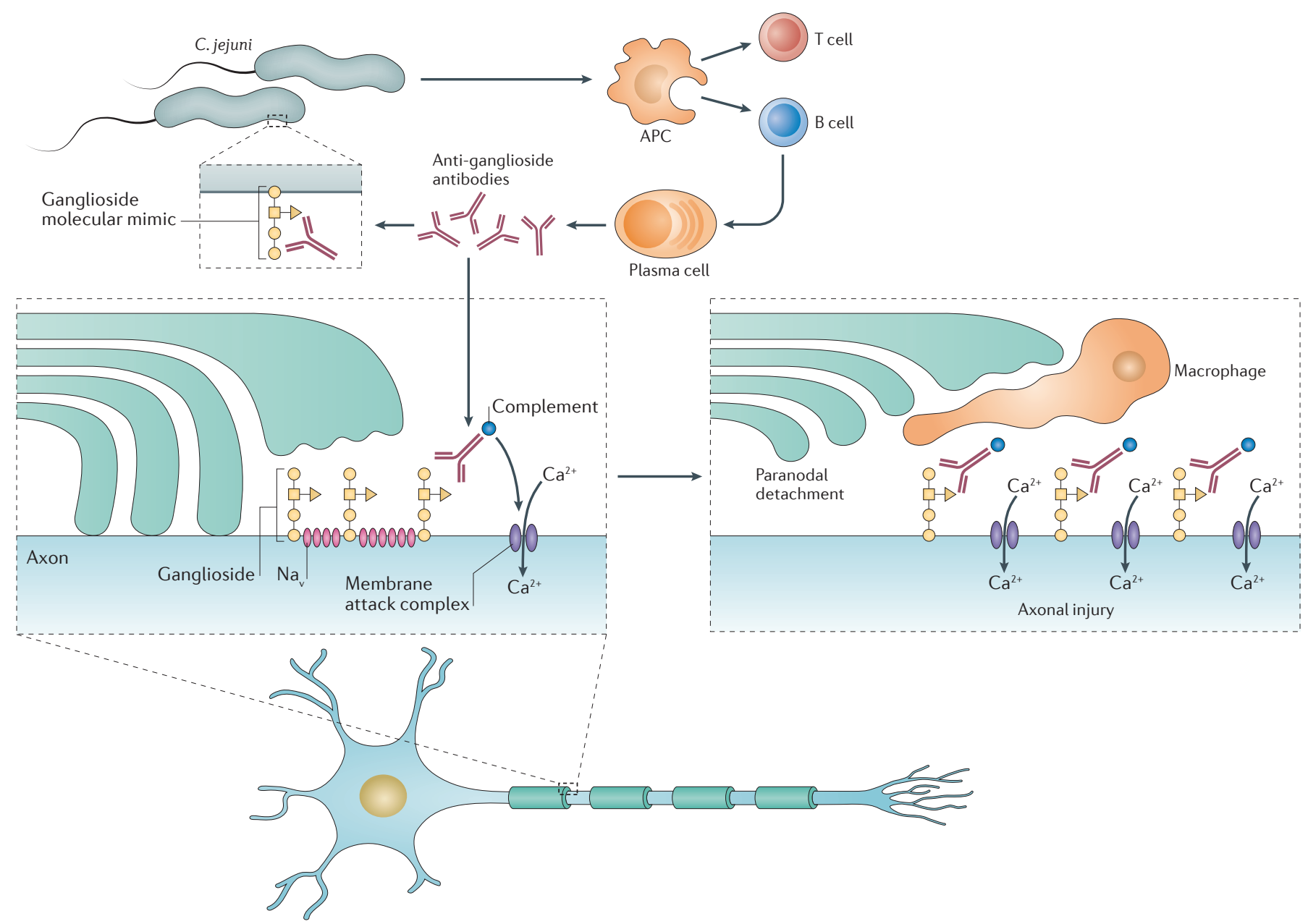

Figure 2 | Proposed mechanism of Guillain-Barré syndrome pathogenesis mediated by Campylobacter jejuni. A molecular mimic of gangliosides in C. jejuni leads to the production of anti-ganglioside antibodies that bind to gangliosides in the axonal membrane at the node of Ranvier. Consequent activation of complement leads to dis- ruption of voltage-gated sodium channel $\left(\mathrm{Na}_{\vee}\right)$ clusters, disruption of the nodal architecture, and formation of the membrane attack complex, which leads to calcium influx. These changes cause axonal injury and attract macrophages, which can then migrate between the axon and myelin. exchange. There was initial debate about whether IVIg was as effective as plasma exchange, and whether treatment-related fluctuations in symptoms in patients who receive IVIg would require re-treatment. However, the consensus became that IVIg is as effective as plasma exchange, and far more straightforward to administer ${ }^{87}$. IVIg has, therefore, become the first-line treatment for GBS in most countries, unless a clinical indication favours plasma exchange, or the expense of IVIg limits its availability in resource-poor settings.

Outcomes of GBS are improved with plasma exchange or IVIg, but many patients remain substantially disabled and experience ongoing fatigue or chronic pain, and mortality remains at $2-3 \%{ }^{88}$. Trials have examined whether combined plasma exchange and IVIg ${ }^{89}$, combined IVIg and steroids ${ }^{90}$ or steroids alone ${ }^{91}$ are more effective treatments. Disappointingly, none of these approaches have been superior to standard therapy in the long term, although a possible minor short-term benefit was seen with intravenous methylprednisone in addition to $\mathrm{IVIg}^{92}$. These negative results leave us with only two partially effective treatments. Currently, a randomized placebo-controlled trial (SID-GBS) is assessing whether GBS patients with a poor prognosis benefit from a second course of IVIg $^{93}$. The effect of a second course of IVIg is also being studied in a nonrandomized fashion in the International GBS Outcome Study (BOX 1).

A growing body of evidence supports the theory that antibody-mediated complement-dependent mechanisms

\section{Box 1 | The International Guillain-Barré Syndrome Outcome Study}

The International Guillain-Barré Syndrome Outcome Study is an international, multicentre, prospective observational study coordinated by B. C. Jacobs at Erasmus MC in Rotterdam, Netherlands. Through this massive international effort, every patient with GBS at participating centres will be entered into an observational study in which clinical, electrophysiological, cerebrospinal fluid, serum, treatment and outcome data are collected in order to define biomarkers for disease activity and recovery, and to develop prognostic models. Recruitment for the study began in May 2012, with an original intention to collect data from 1,000 patients. Already, the target has been surpassed, with $>1,250$ patients from 18 countries included to date, and continues to recruit patients in many countries from all continents throughout the world. 
underlie the pathogenesis of $\mathrm{GBS}^{94}$, and ongoing clinical trials are exploring whether specific complement inhibition can improve outcomes of GBS. One drug currently in trials is eculizumab, an anti-C5 monoclonal antibody that is licensed for the treatment of paroxysmal nocturnal haemoglobinuria. In a mouse model of GBS, eculizumab has been shown to prevent complement-mediated injury ${ }^{95}$, and in a pilot study in patients with multifocal motor neuropathy, it proved safe and tolerable $e^{96}$. At present, eculizumab is being tested in two randomized, blinded, placebo-controlled trials: the Inhibition of Complement Activation in GBS study $(\mathrm{ICA}-\mathrm{GBS})^{97}$ and the Japanese Eculizumab Trial for GBS (JET-GBS) ${ }^{98}$. In both studies, patients receive standard therapy with IVIg or plasma exchange with either eculizumab or placebo. Both trials have now completed and are due to report their results shortly.

\section{Conclusions and outstanding questions}

In the 100 years since the first landmark description of GBS, we have made considerable progress in understanding the nature of the disease and the mechanisms that lead to its development (FIG. 3). In particular, our understanding of the pathology and pathogenesis of AMAN has increased considerably in recent decades, with a detailed picture now emerging of antibody-mediated and complement-mediated disruption of the neuronal membrane. Nevertheless, many uncertainties remain.

The relative contributions of T cells, macrophages and antibodies in AIDP remain unclear, as most patients with this condition remain seronegative for anti-ganglioside antibodies with the use of traditional antibody detection methods. We do not know whether these patients express an as yet unidentified autoantibody, or whether the immunopathogenesis is fundamentally different in this pathological subtype. Similarly, we do not yet know whether complement inhibition will prove to be an effective therapy for GBS and, if so, whether it will benefit only patients with AMAN, in whom there is considerable evidence for the role of complement in pathogenesis, or also patients with AIDP.

One reason GBS has proven difficult to study is that it is a heterogenous disorder, and even interested clinicians and centres

Figure 3 | Timeline showing the major milestones in Guillain-Barré syndrome (GBS). CSF, cerebrospinal fluid; EAN, experimental autoimmune neuritis; IVIg, intravenous immunoglobulin.

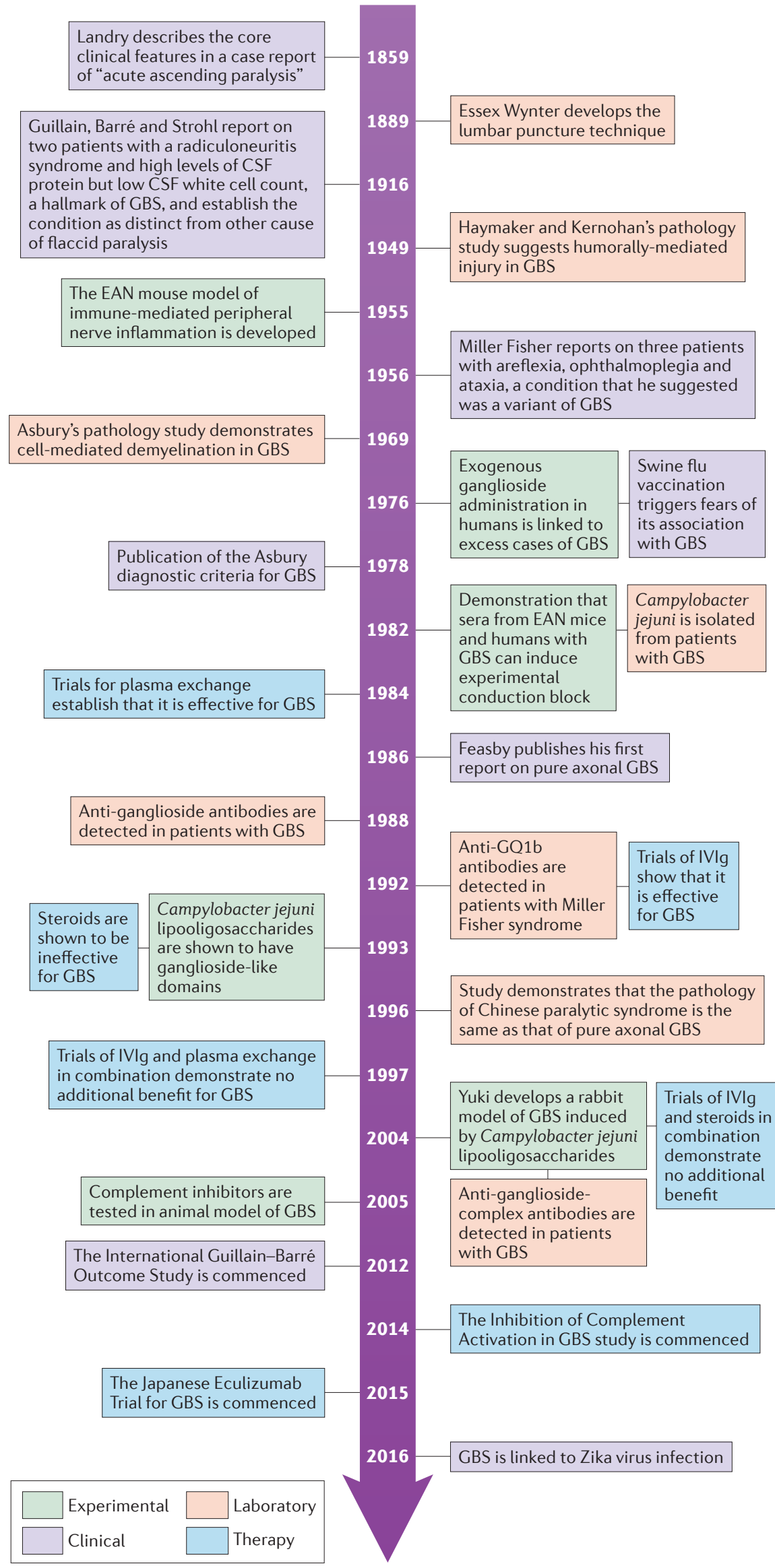

Asbury's pathology study demonstrates
cell-mediated demyelination in GBS

Trials for plasma exchang establish that it is effective for GB

Steroids are
shown to be
ineffective
for GBS Campylobacter jejuni
lipooligosaccharides
are shown to have
ganglioside-like
domains

Trials of IVIg and plasma exchang in combination demonstrate no dditional benefit for GBS
Anti-gangliosidecomplex antibodies are detected in patients with GBS

The Inhibition of Complement Activation in GBS study is commenced

\section{5}


see relatively few cases each year. Global collaboration through initiatives such as the International Guillain-Barré Syndrome Outcome Study (BOX 1) is likely to enable development of biomarkers, prognostic tools and personalized therapy, and provide a clearer understanding of the aetiology and pathogenesis of GBS so that we can answer the remaining, long-standing questions.

In the 100th anniversary year of GBS, a new challenge has also come to the fore in the association of Zika virus infection with GBS. An immediate and pressing question is how countries that are experiencing or anticipating a Zika virus outbreak can prepare for the expected increase in cases of GBS, which threaten to overwhelm hospital and intensive care services. The immediacy of the challenge also highlights the importance of building on our progress so far, and gaining an even greater understanding of GBS in the years to come.

John A. Goodfellow is at the Neuroimmunology Laboratory, Laboratory Building, Queen Elizabeth University Hospital, Glasgow, G51 4TF, Scotland, UK.

Hugh J. Willison is at the Institute of Infection, Immunity and Inflammation, University of Glasgow, University Place, Glasgow, G12 8TA, Scotland, UK.

Correspondence to J.A.G. and H.J.W. jagoodfellow@doctors.org.uk; hugh.willison@glasgow.ac.uk

doi:10.1038/nrneurol.2016.172 Published online 18 Dec 2016

1. Landry, O. Note sur la paralysie ascendante aiguë. Gaz. Hebd. Méd. Chir. 6, 472-474, 486-488 (in French) (1859).

2. Guillain, G., Barrê, J. A. \& Strohl, A. Radiculoneuritis syndrome with hyperalbuminosis of cerebrospinal fluid without cellular reaction. Notes on clinical features and graphs of tendon reflexes. 1916. Ann. Med. Interne (Paris) 150, 24-32 (in French) (1999).

3. Pearce, J. M. Walter Essex Wynter, Quincke, and lumbar puncture. J. Neurol. Neurosurg. Psychiatry 57, 179 (1994).

4. Fokke, C. et al. Diagnosis of Guillain-Barré syndrome and validation of Brighton criteria. Brain 137, 33-43 (2014).

5. Draganescu, S. \& Claudian, J. Sur un cas de radiculonévrite curable (syndrome de Guillain-Barré). Rev. Neurol. 2, 517-521 (in French) (1927).

6. Haymaker, W. E. \& Kernohan, J. W. The LandryGuillain-Barré syndrome; a clinicopathologic report of 50 fatal cases and a critique of the literature. Medicine (Baltimore) 28, 59-141 (1949).

7. Waksman, B. H. \& Adams, R. D. Allergic neuritis: an experimental disease of rabbits induced by the injection of peripheral nervous tissue and adjuvants. J. Exp. Med. 102, 213-236 (1955).

8. Astrom, K. E. $\&$ Waksman, B. H. The passive transfer of experimental allergic encephalomyelitis and neuritis with living lymphoid cells. J. Pathol. Bacteriol. $\mathbf{8 3}$, 89-106 (1962).

9. Kadlubowski, M. \& Hughes, R. A. Identification of the neuritogen for experimental allergic neuritis. Nature 277, 140-141 (1979).

10. Linington, C. et al. A permanent rat $\mathrm{T}$ cell line that mediates experimental allergic neuritis in the Lewis rat in vivo. J. Immunol. 133, 1946-1950 (1984).

11. Yan, W. et al. Antibodies to neurofascin exacerbate adoptive transfer experimental autoimmune neuritis. J. Neuroimmunol. 277, 13-17 (2014).

12. Asbury, A. K., Arnason, B. G. \& Adams, R. D. The inflammatory lesion in idiopathic polyneuritis. Its role in pathogenesis. Medicine (Baltimore) 48, 173-215 (1969).

13. Wišniewski, H., Terry, R. D., Whitaker, J. N., Cook, S. D. \& Dowling, P. C. Landry-Guillain-Barrê syndrome. A primary demyelinating disease. Arch. Neurol. 21 269-276 (1969)

14. Prineas, J. W. Acute idiopathic polyneuritis. An electron microscope study. Lab. Invest. 26, 133-147 (1972)

15. Brechenmacher, C. et al. Guillain-Barré syndrome: an ultrastructural study of peripheral nerve in 65 patients. Clin. Neuropathol 6, 19-24 (1987).

16. Fisher, M. An unusual variant of acute idiopathic polyneuritis (syndrome of ophthalmoplegia, ataxia and areflexia). N. Engl. J. Med. 255, 57-65 (1956)

17. Shahrizaila, N. \& Yuki, N. Bickerstaff brainstem encephalitis and Fisher syndrome: anti-GQ1b antibody syndrome. J. Neurol. Neurosurg. Psychiatry 84, 576-583 (2013).

18. Schonberger, L. B. et al. Guillain-Barre syndrome following vaccination in the National Influenza Immunization Program, United States, 1976-1977. Am. J. Epidemiol. 110, 105-123 (1979).

19. Kurland, L. T., Wiederholt, W. C., Kirkpatrick, J. W., Potter, H. G. \& Armstrong, P. Swine influenza vaccine and Guillain-Barré syndrome. Epidemic or artifact? Arch. Neurol. 42, 1089-1090 (1985).

20. Langmuir, A. D., Bregman, D. J., Kurland, L. T., Nathanson, N. \& Victor, M. An epidemiologic and clinical evaluation of Guillain-Barré syndrome reported in association with the administration of swine influenza vaccines. Am. J. Epidemiol. 119 841-879 (1984).

21. Lasky, T. et al. The Guillain-Barre syndrome and the 1992-1993 and 1993-1994 influenza vaccines. N. Engl. J. Med. 339, 1797-1802 (1998).

22. Osler, L. D. \& Sidell, A. D. The Guillain-Barre syndrome; the need for exact diagnostic criteria. N. Engl. J. Med. 262, 964-969 (1960).

23. [No authors listed.] Criteria for diagnosis of GuillainBarré syndrome. Ann. Neurol. 3, 565-566 (1978).

24. Asbury, A. K. Diagnostic considerations in GuillainBarré syndrome. Ann. Neurol. 9 (Suppl.), 1-5 (1981).

25. Asbury, A. K. \& Cornblath, D. R. Assessment of current diagnostic criteria for Guillain-Barré syndrome. Ann. Neurol. 27 (Suppl.), S21-S24 (1990).

26. Schengrund, C. L. Gangliosides: glycosphingolipids essential for normal neural development and function. Trends Biochem. Sci. 40, 397-406 (2015).

27. Dodge, J. C. et al. Glycosphingolipids are modulators of disease pathogenesis in amyotrophic lateral sclerosis. Proc. Natl Acad. Sci. USA 112, 8100-8105 (2015).

28. Nagai, Y., Momoi, T., Saito, M., Mitsuzawa, E. \& Ohtani, S. Ganglioside syndrome, a new autoimmune neurologic disorder, experimentally induced with brain gangliosides. Neurosci. Lett. 2 107-111 (1976).

29. Díez-Tejedor, E., Gutiérrez-Rivas, E. \& Gil-Peralta, A. Gangliosides and Guillain-Barre syndrome: the Spanish data. Neuroepidemiology 12, 251-256 (1993).

30. Matias-Guiu, J. et al. Incidence of Guillain-Barré syndrome and ganglioside intake in Alcoi, Spain Neuroepidemiology 12, 58-60 (1993).

31. Geisler, F. H., Dorsey, F. C. \& Coleman, W. P. Recovery of motor function after spinal-cord injury - a randomized, placebo-controlled trial with $\mathrm{GM}-1$ ganglioside. N. Engl. J. Med. 324, 1829-1838 (1991).

32. Wiederholt, W., Mulder, D. \& Lambert, E. The Landry-Guillain-Barre-Strohl syndrome or polyradiculoneuropathy: historical review, report on 97 patients, and present concepts. Mayo Clin. Proc. 39, 427-451 (1964).

33. Sumner, A. J., Saida, K., Saida, T., Silberberg, D. H. $\&$ Asbury, A. K. Acute conduction block associated with experimental antiserum-mediated demyelination of peripheral nerve. Ann. Neurol. 11, 469-477 (1982)

34. Lafontaine, S., Rasminsky, M., Saida, T. $\bar{\varnothing}$ Sumner, A. J. Conduction block in rat myelinated fibres following acute exposure to antigalactocerebroside serum. J. Physiol. 323, 287-306 (1982).

35. Saida, T. et al. In vivo demyelinating activity of sera from patients with Guillain-Barré syndrome. Ann. Neurol. 11, 69-75 (1982)

36. Albers, J. W., Donofrio, P. D. \& McGonagle, T. K. Sequential electrodiagnostic abnormalities in acute inflammatory demyelinating polyradiculoneuropathy. Muscle Nerve 8, 528-539 (1985).
37. McLeod, J. G. Electrophysiological studies in the Guillain-Barré syndrome. Ann. Neurol. 9 (Suppl.), 20-27 (1981).

38. Uncini, A. \& Kuwabara, S. Electrodiagnostic criteria for Guillain-Barrè syndrome: a critical revision and the need for an update. Clin. Neurophysiol. 123 1487-1495 (2012).

39. Feasby, T. E. et al. An acute axonal form of Guillain-Barré polyneuropathy. Brain 109 1115-1126 (1986)

40. Feasby, T. E. et al. Acute "axonal" Guillain-Barré polyneuropathy. Neurology 37, 357 (1987).

41. Yuki, N., Yoshino, H., Sato, S. ¿ Miyatake, T. Acute axonal polyneuropathy associated with anti-GM 1 antibodies following Campylobacter enteritis. Neurology 40, 1900-1902 (1990).

42. Hafer-Macko, C. et al. Acute motor axonal neuropathy: an antibody-mediated attack on axolemma. Ann. Neurol. 40, 635-644 (1996).

43. McKhann, G. M. et al. Clinical and electrophysiological aspects of acute paralytic disease of children and young adults in northern China. Lancet 338 , 593-597 (1991)

44. Griffin, J. W. et al. Early nodal changes in the acute motor axonal neuropathy pattern of the Guillain-Barré syndrome. J. Neurocytol. 25, 33-51 (1996).

45. Hafer-Macko, C. E. et al. Immune attack on the Schwann cell surface in acute inflammatory demyelinating polyneuropathy. Ann. Neurol. 39 625-635 (1996)

46. Ho, T. W., McKhann, G. M. \& Griffin, J. W. Human autoimmune neuropathies. Annu. Rev. Neurosci. 21 187-226 (1998).

47. Campbell, A. The aetiology of polyneuritis. Proc. R. Soc. Med. 51, 157-159 (1958).

48. Rhodes, K. M. ¿ Tattersfield, A. E. Guillain-Barré syndrome associated with Campylobacter infection Br. Med. J. (Clin. Res. Ed.) 285, 173-174 (1982).

49. Kaldor, J. \& Speed, B. R. Guillain-Barré syndrome and Campylobacter jejuni: a serological study. Br. Med. J. (Clin. Res. Ed.) 288, 1867-1870 (1984).

50. Rees, J. H., Soudain, S. E., Gregson, N. A. \& Hughes, R. A. Campylobacter jejuni infection and Guillain-Barré syndrome. N. Engl. J. Med. 333 1374-1379 (1995).

51. Yuki, N. et al. A bacterium lipopolysaccharide that elicits Guillain-Barré syndrome has a GM1 ganglioside-like structure. J. Exp. Med. 178 1771-1775 (1993).

52. Yuki, N. et al. Molecular mimicry between $\mathrm{GQ} 1 \mathrm{~b}$ ganglioside and lipopolysaccharides of Campylobacter jejuni isolated from patients with Fisher's syndrome. Ann. Neurol. 36, 791-793 (1994).

53. Yuki, N. et al. Carbohydrate mimicry between human ganglioside GM1 and Campylobacter jejuni lipooligosaccharide causes Guillain-Barrê syndrome. Proc. Natl Acad. Sci. USA 101, 11404-11409 (2004)

54. Ilyas, A. A. et al. Serum antibodies to gangliosides in Guillain-Barré syndrome. Ann. Neurol. 23, 440-447 (1988).

55. Gregson, N. A., Jones, D., Thomas, P. K. \& Willison, H. J. Acute motor neuropathy with antibodies to GM1 ganglioside. J. Neurol. 238, 447-451 (1991).

56. Ho, T. W. et al. Anti-GD1 a antibody is associated with axonal but not demyelinating forms of Guillain-Barré syndrome. Ann. Neurol. 45, 168-173 (1999).

57. O'Leary, C. P. et al. Acute oropharyngeal palsy is associated with antibodies to GQ1b and GT $1 \mathrm{a}$ gangliosides. J. Neurol. Neurosurg. Psychiatry 61, 649-651 (1996)

58. Chiba, A., Kusunoki, S., Shimizu, T. \& Kanazawa, I. Serum IgG antibody to ganglioside $\mathrm{GQ} 1 \mathrm{~b}$ is a possible marker of Miller Fisher syndrome. Ann. Neurol. 31, 677-679 (1992)

59. Kaida, K. et al. Ganglioside complexes as new target antigens in Guillain-Barré syndrome. Ann. Neurol. 56 567-571 (2004)

60. Pestronk, A., Choksi, R., Blume, G. \& Lopate, G Multifocal motor neuropathy: serum IgM binding to a GM1 ganglioside-containing lipid mixture but not to GM1 alone. Neurology 48, 1104-1106 (1997).

61. Rinaldi, S. et al. Antibodies to heteromeric glycolipid complexes in Guillain-Barré syndrome. PLOS ONE 8, e82337 (2013).

62. Rinaldi, S., Brennan, K. M. \& Willison, H. J. Heteromeric glycolipid complexes as modulators of autoantibody and lectin binding. Prog. Lipid Res. 49, 87-95 (2010). 
63. Rinaldi, S., Brennan, K. M. \& Willison, H. J. Combinatorial glycoarray. Methods Mol. Biol. $\mathbf{8 0 8}$, 413-423 (2012)

64. Willison, H. J. et al. Mechanisms of action of anti-GM1 and anti-GQ1b ganglioside antibodies in GuillainBarré syndrome. J. Infect. Dis. 176 (Suppl. 2), S144-S149 (1997).

65. Greenshields, K. N. et al. The neuropathic potential of anti-GM 1 autoantibodies is regulated by the local glycolipid environment in mice. J. Clin. Invest. 119 , 595-610 (2009).

66. Chiba, A., Kusunoki, S., Obata, H., Machinami, R. ¿ Kanazawa, I. Serum anti-GQ1 1 IgG antibody is associated with ophthalmoplegia in Miller Fisher syndrome and Guillain-Barré syndrome: clinical and immunohistochemical studies. Neurology 43, 1911-1917 (1993).

67. Goodfellow, J. A. et al. Overexpression of GD1a ganglioside sensitizes motor nerve terminals to anti-GD 1 a antibody-mediated injury in a model of acute motor axonal neuropathy. J. Neurosci. 25, 1620-1628 (2005).

68. McGonigal, R. et al. Anti-GD1 a antibodies activate complement and calpain to injure distal motor nodes of Ranvier in mice. Brain 133, 1944-1960 (2010).

69. Susuki, K. et al. Anti-GM1 antibodies cause complement-mediated disruption of sodium channel clusters in peripheral motor nerve fibers. J. Neurosci. 27, 3956-3967 (2007)

70. Halstead, S. K. et al. Anti-disialoside antibodies kill perisynaptic Schwann cells and damage motor nerve terminals via membrane attack complex in a murine model of neuropathy. Brain 127, 2109-2123 (2004).

71. O'Hanlon, G. M. et al. Anti-GQ1 b ganglioside antibodies mediate complement-dependent destruction of the motor nerve terminal. Brain 124, 893-906 (2001).

72. Halstead, S. K. et al. Complement inhibition abrogates nerve terminal injury in Miller Fisher syndrome. Ann. Neurol. 58, 203-210 (2005).

73. McGonigal, R et al. C1 q-targeted inhibition of the classical complement pathway prevents injury in a novel mouse model of acute motor axonal neuropathy. Acta Neuropathol. Commun. 4, 23 (2016).

74. Kusunoki, S., Shiina, M. \& Kanazawa, I. Anti-Gal-C antibodies in GBS subsequent to mycoplasma infection: evidence of molecular mimicry. Neurology 57, 736-738 (2001)

75. Cao-Lormeau, V. M. et al. Guillain-Barré Syndrome outbreak associated with Zika virus infection in French Polynesia: a case-control study. Lancet 387 1531-1539 (2016).

76. Araujo, L. M., Ferreira, M. L. \& Nascimento, O. J. Guillain-Barrê syndrome associated with the Zika virus outbreak in Brazil. Arq. Neuropsiquiatr. 74 253-255 (2016)
77. White, M. K., Wollebo, H. S., David Beckham, J. Tyler, K. L. \& Khalili, K. Zika virus: an emergent neuropathological agent. Ann. Neurol. 80, 479-489 (2016).

78. Parra, B. et al. Guillain-Barré syndrome associated with Zika virus infection in Colombia. N. Engl. J. Med. 375, 1513-1523 (2016).

79. Greenwood, R. J. et al. Controlled trial of plasma exchange in acute inflammatory polyradiculoneuropathy. Lancet 1, 877-879 (1984)

80. Osterman, P. O. et al. Beneficial effects of plasma exchange in acute inflammatory polyradiculoneuropathy. Lancet 2, 1296-1299 (1984).

81. [No authors listed.] Plasmapheresis and acute Guillain-Barrê syndrome. The Guillain-Barrê syndrome Study Group. Neurology 35, 1096-1104 (1985)

82. [No authors listed.] Efficiency of plasma exchange in Guillain-Barré syndrome: role of replacement fluids. French Cooperative Group on Plasma Exchange in Guillain-Barré syndrome. Ann. Neurol. 22, 753-761 (1987)

83. van Doorn, P. A., Brand, A., Strengers, P. F., Meulstee, J. \& Vermeulen, M. High-dose intravenous immunoglobulin treatment in chronic inflammatory demyelinating polyneuropathy: a double-blind, placebo-controlled, crossover study. Neurology 40, 209-212 (1990)

84. Kleyweg, R. P., van der Meché, F. G. \& Meulstee, J. Treatment of Guillain-Barrê syndrome with high-dose gammaglobulin. Neurology 38, 1639-1641 (1988).

85. Vermeulen, M., van der Meché, F. G., Speelman, J. D., Weber, A. \& Busch, H. F. Plasma and gamma-globulin infusion in chronic inflammatory polyneuropathy. J. Neurol. Sci. 70, 317-326 (1985).

86. van der Meché, F. G. \& Schmitz, P. I. A randomized trial comparing intravenous immune globulin and plasma exchange in Guillain-Barré syndrome. Dutch Guillain-Barré Study Group. N. Engl. J. Med. 326, 1123-1129 (1992)

87. Hughes, R. A., Swan, A. V. \& van Doorn, P. A. Intravenous immunoglobulin for Guillain-Barré syndrome. Cochrane Database Syst. Rev. 9 , CD002063 (2014).

88. van den Berg, B., Bunschoten, C., van Doorn, P. A $\&$ Jacobs, B. C. Mortality in Guillain-Barre syndrome. Neurology 80, 1650-1654 (2013).

89. [No authors listed.] Randomised trial of plasma exchange, intravenous immunoglobulin, and combined treatments in Guillain-Barré syndrome. Plasma Exchange/Sandoglobulin Guillain-Barré Syndrome Trial Group. Lancet 349, 225-230 (1997).

90. van Koningsveld, R. et al. Effect of methylprednisolone when added to standard treatment with intravenous immunoglobulin for Guillain-Barrē syndrome: randomised trial. Lancet 363, 192-196 (2004).

91. [No authors listed.] Double-blind trial of intravenous methylprednisolone in Guillain-Barré syndrome. Guillain-Barré Syndrome Steroid Trial Group. Lancet 341, 586-590 (1993).

92. Hughes, R. A. \& van Doorn, P. A. Corticosteroids for Guillain-Barré syndrome. Cochrane Database Syst. Rev. 8, CD001446 (2012).

93. Netherlands Trial Register. trialregister. $n /$ http://www. trialregister.nl/trialreg/admin/rctview.asp? $\mathrm{TC}=2224$ (2016)

94. Plomp, J. J. \& Willison, H. J. Pathophysiologica actions of neuropathy-related anti-ganglioside antibodies at the neuromuscular junction. J. Physiol. 587, 3979-3999 (2009).

95. Halstead, S. K. et al. Eculizumab prevents anti-ganglioside antibody-mediated neuropathy in a murine model. Brain 131, 1197-1208 (2008).

96. Fitzpatrick, A. M. et al. An open label clinical trial of complement inhibition in multifocal motor neuropathy J. Peripher. Nerv. Syst. 16, 84-91 (2011).

97. US National Library of Medicine. ClinicalTrials.gov https://clinicaltrials.gov/ct2/show/NCT02029378 (2014).

98. US National Library of Medicine. ClinicalTrials.gov https://clinicaltrials.gov/ct2/show/NCT02493725 (2016).

Acknowledgements

H.J.W. receives Wellcome Trust funding for research into Guillain-Barrē syndrome.

\section{Author contributions}

The authors contributed equally to all aspects of the manuscript.

\section{Competing interests statement}

The authors declare no competing interests.

\section{FURTHER INFORMATION}

The Brighton Collaboration: www.brightoncollaboration.org International Guillain-Barré Outcome Study:

https://gbsstudies.erasmusmc.nl/home

To celebrate the progress made in the 100 years since Guillain, Barré and Strohl's case report, an international symposium was held in Glasgow, UK, in June 2016. To mark the event, the Peripheral Nerve Society published a free multi-author textbook summarizing the landmark studies and events in the history of GBS research. This book is freely available as an e-book from www.pnsociety.com.

ALL LINKS ARE ACTIVE IN THE ONLINE PDF 